\title{
Interactions of afterimages for orientation and color: Experimental data and model simulations
}

\author{
GREGORY FRANCIS and MARK ROTHMAYER \\ Purdue University, West Lafayette, Indiana
}

\begin{abstract}
Sequential viewing of two orthogonally related patterns produces an afterimage of the first pattern. We report an experiment that quantifies some properties of this type of afterimage. It is shown that it is important for the two patterns to have orthogonal orientations and that the appearance of the afterimage does not depend on the spatial frequency of the second pattern. We then show that Grossberg's model of interacting boundary and feature contour systems can account for the observed properties of these afterimages.
\end{abstract}

Vidyasagar, Buzas, Kisyarday, and Eysel (1999) noted that viewing a sequence of inducing images produced a visual percept related to the previously seen image. Figure 1a schematizes the sequence of images used by Vidyasagar et al. The sequence consisted of four frames of images that cycled repeatedly. The first image consisted of black and white radial arcs. The second image was a blank gray screen. The third image was a set of black and white concentric circles. The fourth image was another blank gray screen. The arcs and circles moved during their presentation. The arcs rotated clockwise and then counterclockwise; the circles converged and then diverged relative to the image center.

The percepts associated with this sequence of images are schematized in Figure 1b. During the presentation of the arcs or circles, observers veridically saw those images. During the presentation of a gray screen, observers reported seeing a percept related to the inducing stimulus from three frames back. Thus, offset of the arcs image produced an afterimage of nonmoving black and white concentric circles, whereas offset of the concentric circles image produced an afterimage of nonmoving black and white arcs. The schematic in Figure $1 \mathrm{~b}$ may exaggerate the clarity of the effect somewhat, since there are often regions in the afterimage that seem to fade into the gray background. Nevertheless, the existence and the shape of the afterimage were quite evident.

It is not entirely clear how this afterimage compares with other types of afterimages and aftereffects. As suggested by the observations of Vidyasagar et al. (1999) and

G.F. was supported by a fellowship at the Hanse Wissenschaftskolleg, Delmenhorst, Germany. Correspondence should be addressed to G. Francis, Department of Psychological Sciences, 1364 Psychological Sciences Building, Purdue University, West Lafayette, IN 47907-1364 (email: gfrancis@psych.purdue.edu). verified by an experiment discussed below, the appearance of the afterimage seems to generally require the sequential presentation of two properly oriented inducers. The traditional retinal negative afterimage requires only one inducing stimulus. Nevertheless, the inducing stimuli used by Vidyasagar et al. produce an afterimage percept that is visible on a blank background. The ability to be observed on a blank background distinguishes this type of afterimage from a number of aftereffects involving tilt and curvature (Gibson, 1933), figural shape (Köhler \& Wallach, 1944), spatial frequency (Blakemore \& Sutton, 1969), and various contingencies (e.g., McCollough, 1965).

The new afterimage does seem to bear similarities to the orientation afterimages that were popularized by MacKay (1957). An orientation afterimage is seen after an observer has stared at a regular oriented pattern (e.g., a set of concentric circles). At offset of the inducer pattern, the observer sees shimmering wavy lines that run locally orthogonal to the orientations of the inducer pattern. These kinds of afterimages can be created with many types of stimuli; fixation of a pattern consisting of radial arcs produces an afterimage of circles, and fixation of horizontal lines leads to an afterimage of vertical lines and vice versa. As with the afterimage observed by Vidyasagar et al. (1999), it is not clear whether the MacKay afterimages should be called afterimages or aftereffects. The classification is difficult because, unlike the afterimage noted by Vidyasagar et al., the MacKay afterimages do not seem to include a percept of color or brightness. Instead, the percept is of streaming lines of an orthogonal orientation. Thus, although something is clearly seen at offset of the inducing stimulus, it is not an afterimage in the sense that that term is generally used. MacKay referred to these percepts as complementary afterimages (CAIs) to indicate that the shape of the afterimage is, in some sense, the orthogonal complement of the inducing image. We use this nomenclature in our discussion. 
(a) Stimuli
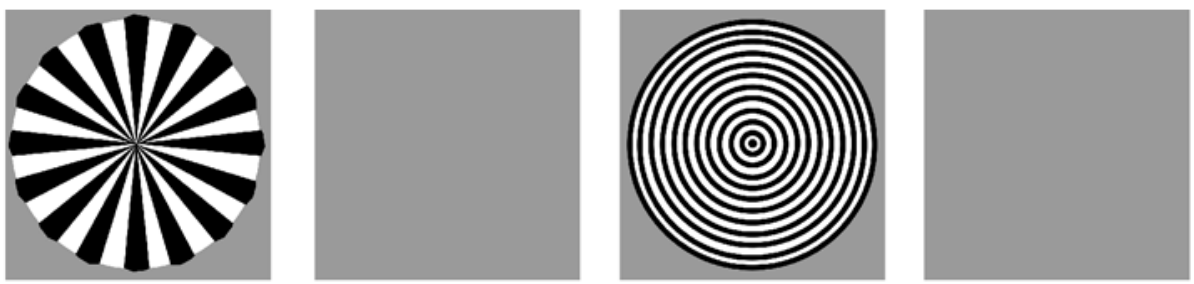

(b) Percepts
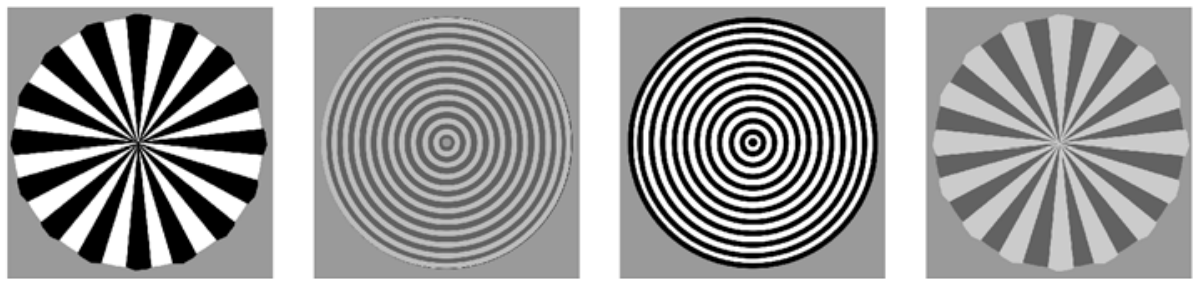

Figure 1. A schematic of the experiment from Vidyasagar et al. (1999). (a) The sequence of images from left to right follows the order of presentation. The sequence cycled repeatedly. The arcs image rotated clockwise and counterclockwise, and the circles image contracted and expanded. (b) The percepts of observers. During the blank gray images, observers reported seeing a faint nonmoving afterimage that was related to the image presented three frames back.

We feel that a relationship between MacKay's (1957) CAIs and the new afterimage observed by Vidyasagar et al. (1999) can be appreciated by classifying the afterimages as modal or amodal percepts (Kanizsa, 1979). Modal percepts include a phenomenologicalpresence; such percepts can be either real or illusory contours. Amodal percepts do not include a phenomenological presence. A common example of an amodal percept is an awareness of an object that is partially occluded by another object. The properties of the occluded object are not seen directly, but they are still perceived. CAIs include knowledge of shape and orientation but seem to be devoid of color and do not seem to include a surface. Thus, the CAI percepts could be classified as amodal afterimages. On the other hand, the Vidyasagar et al. afterimage does include a characterization of colors and surfaces and thus produces a modal afterimage. We suggest (and provide a theory to identify mechanisms) that the Vidyasagar et al. afterimage and MacKay's CAIs are closely related in that the Vidyasagar et al. methodology provides a means of allowing the CAI to capture surface and color information to produce a modal afterimage percept in the normally amodal CAI. In the discussion below, we refer to the afterimage that was observed by Vidyasagar et al. as a modal CAI, or an MCAI.

We support this argument by expanding on the Vidyasagar et al. (1999) findings in two ways. First, we describe an experiment that quantifies the observations of Vidyasagar et al. and demonstrates that the properties of the second stimulus are critical for creation of the MCAI percept. Second, we demonstrate how the neural network model of visual perception proposed by Grossberg and Mingolla (1985a, 1985b) accounts for the properties of these afterimages. We conclude that the characteristics of this afterimage strongly support a distinction between boundary computations and surface filling-in of brightness percepts.

\section{EXPERIMENT}

This experiment serves multiple purposes. First, it provides a quantitative measurement of the MCAI percept. The report of Vidyasagar et al. (1999) was largely anecdotal, with no measurements of behavior. Thus, this experiment provides the first rigorous exploration of the magnitude of the MCAI. Second, it demonstrates that MCAI percepts can be generated with types of stimuli other than those used by Vidyasagar et al. Third, it highlights the importance of the properties of the second stimulus. A Web-based version of the experiment is observable at http:// www.psych.purdue.edu/ gfrancis/Experiments/MCAIs. Since conditions will vary from the laboratory where the data was gathered, the Web-based version may differ slightly from what observers saw during the experiment.

\section{Method}

Figure 2 schematizes the experimental method used in each trial. The observer stared at a small red square at the center of a horizontal bar grating for $1 \mathrm{sec}$. The horizontal bar grating was then replaced by a second stimulus, which varied from trial to trial. In Figure 2 the second stimulus is a vertical bar grating. The second stimulus flickered with its color complement for a total of $1 \mathrm{sec}$ and was then replaced by a blank screen for another second. The end of the trial was marked by a box filled with random dots. The observer's task was to report what, if any, afterimage was seen just before the random dots appeared. 

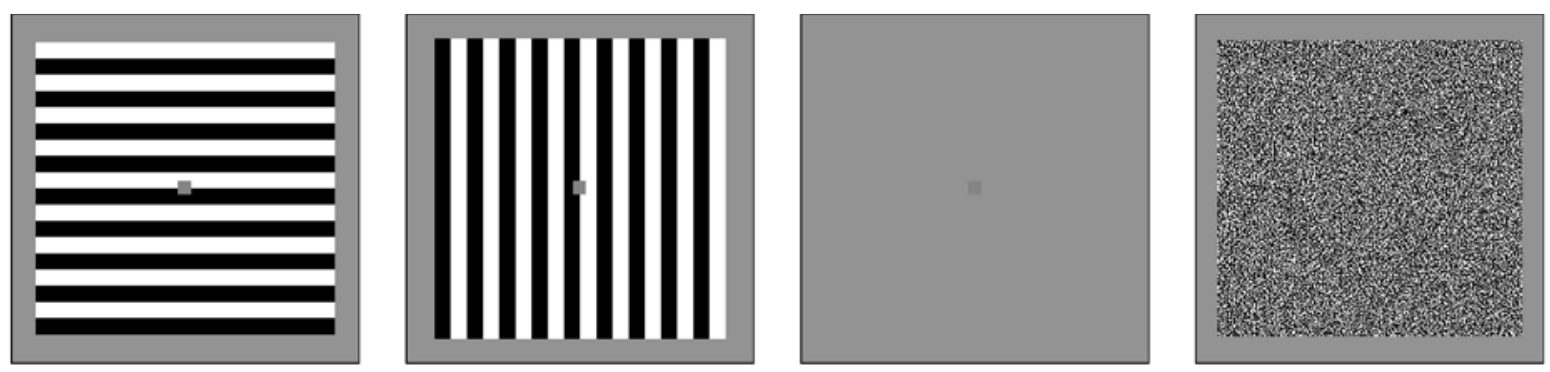

(Flickering)

Figure 2. A schematic of a trial in the experiment. The observer saw a horizontal grating for 1 sec. This was replaced by a second stimulus (in this case a vertical grating) that flickered with its color complement for a total of 1 sec. The second stimulus was replaced by a blank gray screen for 1 sec. Finally, a box filled with random dots was presented. The observer was to report on any afterimages that were seen just before the box of random dots appeared.

All stimuli were presented on a Silicon Graphics Indy computer. The gratings consisted of horizontal or vertical black $\left(0.06 \mathrm{~cd} / \mathrm{m}^{2}\right)$ and white $\left(47 \mathrm{~cd} / \mathrm{m}^{2}\right)$ bars on a gray $\left(41 \mathrm{~cd} / \mathrm{m}^{2}\right)$ background. Each bar in a grating was 460 pixels long (approximately $7.5 \mathrm{deg}$ of visual arc). The first stimulus was always horizontal and consisted of 20 black and white bars that were each 23 pixels wide (approximately 23 min of visual arc).

There were seven types of second stimuli. A blank stimulus was a gray screen, the same intensity as the background. The other stimuli were horizontal or vertical gratings that varied in bar width. A medium bar grating had 20 bars the same width as the first stimulus. A thin bar grating had 40 bars, each 12 pixels wide (approximately 12 min of visual arc). A thick bar grating had 10 bars, each 46 pixels wide (approximately $45 \mathrm{~min}$ of visual arc). During flicker of the second stimulus, each version was presented for $100 \mathrm{msec}$ five times to fill a total 1-sec presentation. Each pixel of the random dot box was either black or white, as determined by a random number generator. When the random dots appeared, they remained on the screen until the start of the next trial.

Seven naive observers were recruited from the experimental subject pool at Purdue University. The observers received course credit for participation in the experiment. Each observer was run separately in a room that, except for the computer monitor, was dark. The observer sat approximately $80 \mathrm{~cm}$ from the computer monitor and was advised to stay in the same position throughout the experiment.

\section{Procedure}

The observer started each trial by a keypress. After the stimuli were presented, the observer indicated, by an additional keypress, what type of afterimage had been seen just before the box of random dots was presented. The choices available to observers were that they saw "nothing," an afterimage that was predominately "horizontal," or an afterimage that was predominately "vertical." The observer was advised to report "nothing" only if the percept was of a blank gray screen. If observers saw any kind of afterimage, they were advised to choose whether it was better characterized as horizontal or vertical.

The first seven trials were practice trials that went through each of the second stimulus types. During the practice trials the observer was introduced to the stimuli and the task and was shown how to enter responses. Data from the practice trials are not included in the results. Including the practice trials, each second stimulus type was used nine times, for a total of 63 trials. The experiment was selfpaced and took between 30 and 45 min to complete.

To try to avoid across-trial effects, a 15-sec delay was introduced between trials. During this delay the box of random dots remained on the screen for $10 \mathrm{sec}$ and then was replaced by the small fixation box for $5 \mathrm{sec}$. Observers were told that they did not have to look at the computer screen during the intertrial delay.

\section{Results}

Figure 3 plots the percentage of times observers reported seeing nothing, horizontal, or vertical afterimage percepts for the different second stimulus types. The percentage of responses for each second stimulus type is combined across observers and is based on 56 trials. Vertical afterimages were almost never reported. Indeed, for four of the seven second stimulus types, there was not a single report of a vertical afterimage for any of the 7 observers.

There was a notable difference between the horizontal and vertical second stimulus types. When the second stimulus consisted of horizontal bars, the reported percept was predominately "nothing." On the other hand, when the second stimulus consisted of vertical bars, the reported percept was predominately "horizontal," which is the MCAI percept. Thus, the MCAI percept depends on the relative orientation of the first and second stimuli (in a less formal experiment we noted that if the first stimulus consisted of vertical bars, the second stimulus needed to consist of horizontal bars to produce a vertical MCAI). The MCAI appears when the first and second frames are oppositely oriented.

The thickness of the bars of the second stimulus have very little effect on the afterimage percept. For the vertical bars, the predominant percept is horizontal, regardless of the thickness of the bars. For the horizontal bars, the predominant percept is nothing, regardless of the thickness of the bars.

In the blank condition, the offset of the first grating was followed by a blank screen that lasted $2 \mathrm{sec}$ before requiring a report. About one third of the time, observers reported seeing a horizontal afterimage with the blank second stimulus. There was substantial variability across the observers. Three of the 7 observers always reported seeing nothing for this condition, whereas 3 other observers reported seeing a horizontal afterimage at least $50 \%$ of the time.

\section{Discussion}

The MCAI percepts that were observed by Vidyasagar et al. (1999) have been found and quantified in single-trial presentations and with different stimulus conditions. It is not necessary to repeatedly cycle the stimuli (as was done 


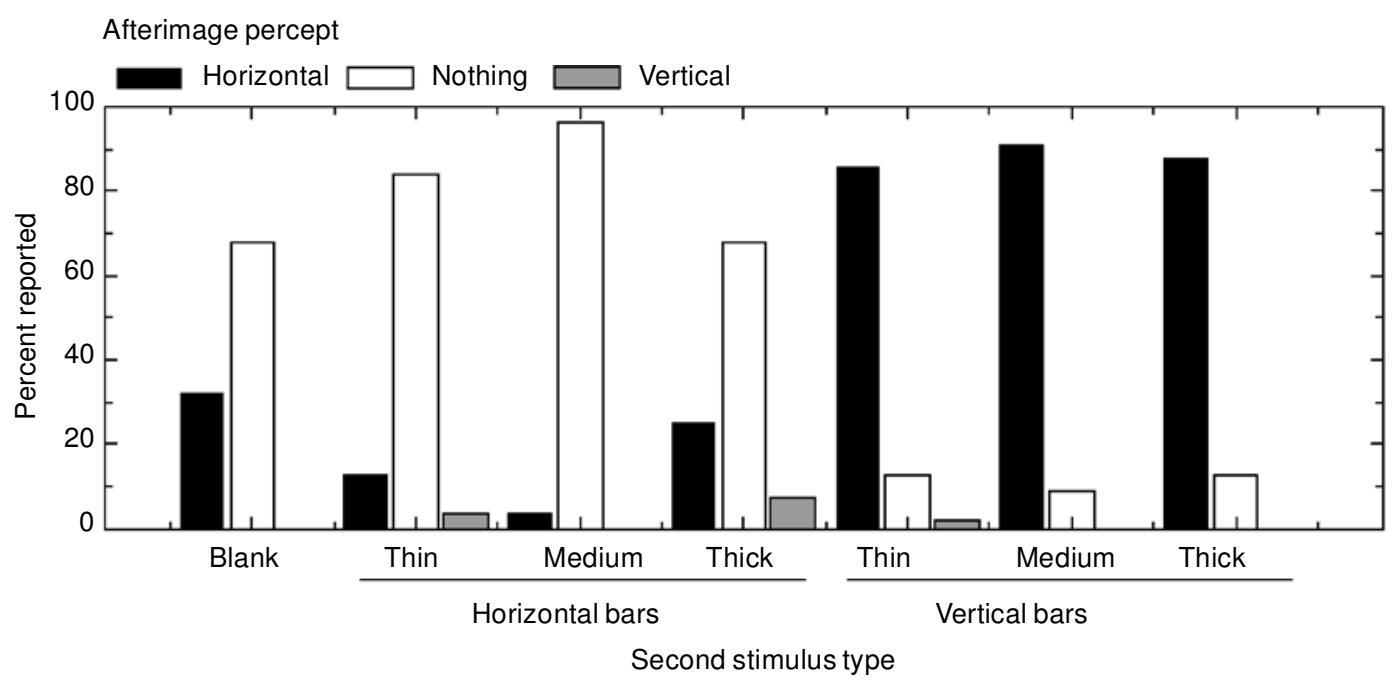

Figure 3. The percentage of reports as a function of the type of second stimulus.

by Vidyasagar et al.), nor is a blank between the first and second stimulus necessary.

At least over the range of values we used, the spatial frequency or bar thickness of the second grating seems to have little effect on the MCAI percept. On the other hand, the orientation of the second grating is very important. The MCAI percept seems to appear only when the second grating is orthogonal to the first.

The differences in reports for the blank condition across observers probably reflect variations in the sensitivity of observers to seeing retinal negative afterimages. One cannot rule out the possibility that retinal negative afterimages are contributing to the percentage of horizontal afterimage percepts when the second stimulus was a vertical grating. However, retinal negative afterimages cannot account for the increased frequency of horizontal afterimage reports when the second stimulus was vertical relative to the blank condition. Moreover, it is significant that the percentage of horizontal reports decreases, relative to the blank condition, when the second stimulus was a flickering horizontal grating. Such a stimulus does not seem to introduce an alternative afterimage, but rather increases the number of "nothing" reports and decreases the number of "horizontal" reports.

An explanation of the experimental results requires a theory that can account for four main effects: (1) Presentation of a horizontal and then a vertical grating produces an afterimage of a horizontal grating; (2) presentation of a horizontal and then another horizontal grating does not produce any kind of afterimage; (3) the above-mentioned effects are insensitive to variations in the spatial frequency of the second grating; and (4) the appearance of the MCAI is not dependent on the appearance of a retinal negative afterimage.

Vidyasagar et al. (1999) proposed that the first inducing stimulus generated a trace that corresponded to the pattern of the inducing stimulus. This trace would include a representation of the orientations of the inducing stimu- lus. The trace was then brought to perceptual threshold by a rebound from inhibition at the offset of the second inducing stimulus. Further, Vidyasagar et al. suggested that the antagonism that underlies the inhibition between orthogonal orientation columns involved competitions between pattern and movement channels.

We agree with the general concepts of Vidyasagar et al. (1999) except for their suggestion of the involvement of movement channels. The stimuli in our experiment did not produce percepts of movement, yet the afterimages were quite strong. This suggests that antagonism between pattern and movement channels is not the key mechanism responsible for the MCAI percept. Further, although we agree with Vidyasagar et al. regarding the existence of a trace and the role of rebounds from inhibition in revealing that trace, we feel that an adequate explanation must describe what types of mechanistic details are necessary to produce the desired effects. We provide such details in the next section. Though our explanation includes the general ideas of Vidyasagar et al., those details are not new features of the model. Moreover, we find that many other characteristics of the model also play critical roles in accounting for the existence of MCAIs.

\section{FACADE THEORY}

The theory that we believe can account for MCAIs is called FACADE, which stands for Form And Color And DEpth (Grossberg, 1994). FACADE is an extension of a model proposed by Grossberg and Mingolla (1985a, $1985 b$ ). Most of the mechanisms we will use are part of the earlier model, but some of the terms introduced in the more general FACADE theory are relevant to our explanation. FACADE is a complex model and its full description is beyond the discussion of this manuscript. Interested readers should look to Grossberg $(1994,1997)$ for discussions of the model to those dates. Only those parts of the 
model that are crucial for accounting for MCAIs are discussed fully, and the simulations are also simplified to include only the most relevant parts.

In the theory proposed by Grossberg and Mingolla (1985a, 1985b), two separate pathways are used to compute visual information. A boundary contour system (BCS) processes edge, or boundary, information without direct regard to the color or brightness of corresponding surfaces. A feature contour system (FCS) processes edge information that retains relative information about surface colors and brightness and also provides stages for fillingin of that information to identify the color and brightness of surfaces. The BCS is concerned with identifying the location and orientation of edgelike information. The FCS uses the layout of BCS boundary information to define the spread of surface information. A set of connected BCS signals can define a closed region called a Filling-In DOmain, or FIDO (Grossberg, 1994). The FCS signals that are spatially located within a FIDO are constrained to spread information only within the FIDO. Separate FIDOs correspond to surface regions with different perceived brightness or color.

Figure 4 schematizes the minimal components of FACADE that can account for the properties of MCAIs. Not all connections and interactions are drawn in this schematic representation, and for simplicity the discussion is restricted to achromatic colors. The input image projects first to a pixel representation of the black and white components of the image. The dual arrow between the black cells and white cells represents a type of competition that is described in more detail below. The black and white representation then projects to two parallel processing systems. The BCS detects oriented edges, and the vertical and horizontal ellipses are meant to indicate the cells that code vertical and horizontal orientations. The dual arrow between the vertical and horizontal cells represents a type of competition that is described in more detail below. The dual pointed arrows between the lower and higher levels of the BCS indicate a cooperative-competitive loop that uses feedback to complete broken contours and select the best representation of oriented edges.

The FCS contains separate filling-in stages for black and white percepts. Each cell in a filling-in stage shares information with its neighbors, unless BCS signals block them. This spreading of information corresponds to a hypothesized filling-in process that underlies the percepts of surface brightness and color. Although more elaborated interactions between the BCS and FCS are described in Grossberg (1994, 1997), for the present simulations the activities of cells across the filling-in stage correspond to the brightness percept. The spread of information can be blocked by the presence of appropriate BCS signals. This interaction is schematized by the arrow feeding into the gray rounded-corner square around the center cell of each filling-in stage.

Figure 5a shows simulation results from different parts of the BCS and FCS in response to presentation of a horizontal grating. Most of the simulation results will be presented in this format. Details of the simulations and model

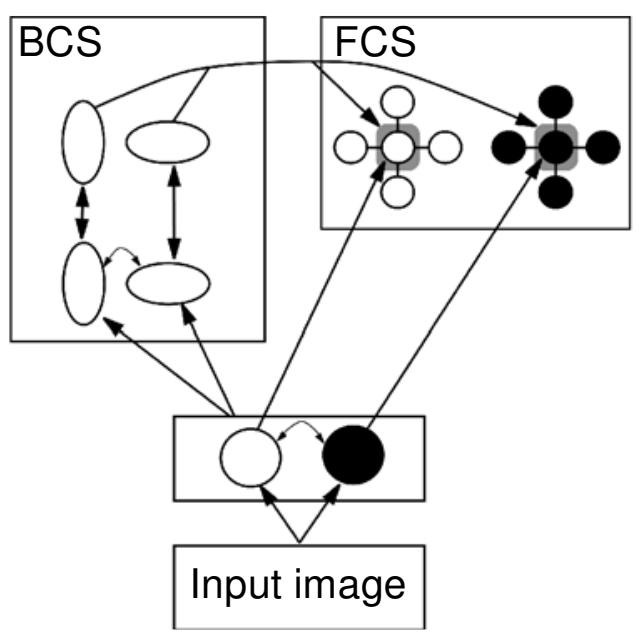

Figure 4. A schematic of the main components of FACADE theory that are relevant for MCAIs. The input image feeds into a retinotopic representation of black and white. The black and white information then feeds separately into edge detection in the BCS and a filling-in stage in the FCS. The edges in the BCS also influence the filling-in stage to limit the spread of color and brightness information.

equations are provided in the Appendix. Each frame plots values across all the pixels from one level of the model. Each frame combines information from two sources (e.g., black and white colors or horizontal and vertical orientations) by subtracting the simulation value of one component from the other at each pixel. The resulting difference of values is plotted with an intensity that codes the relative value of activity. This is done by always setting the value zero as middle gray. Positive values are then plotted as lighter grays and negative values are plotted as darker grays. For each plot, the simulated values are normalized and then mapped to gray levels. Thus, the extrema of each set of difference values will be set to black (if negative) or white (if positive).

Above or below each frame is a symbol or phrase taken from Figure 4 to indicate what model-level activity is being plotted. The lower left frame is the input image. The lower right frame corresponds to an early model representation of black and white. The upper left frame corresponds to the representation of vertical and horizontal orientations. The upper right frame corresponds to the black and white filling-in stages. These are discussed in detail below.

The bar grating in the lower left of Figure 5a represents the input image, in this case a black-and-white striped horizontal grating on a gray background. The frame on the bottom right shows the activation of the cells tuned to the presence of white and black in the input image. At each pixel location, middle gray indicates that neither a white nor black cell is responding. Light gray and white indicates activity from a cell tuned to the presence of white in the image. Dark gray and black indicates activity from a cell tuned to the presence of black in the image. The net result is that this frame looks like a replication of the input 
(a)
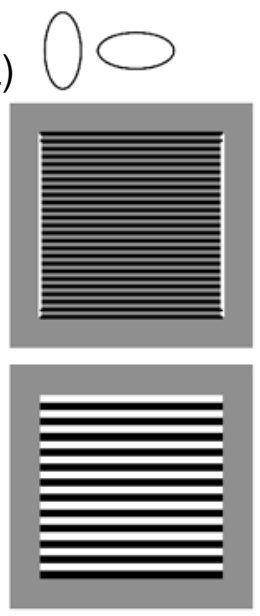

Image
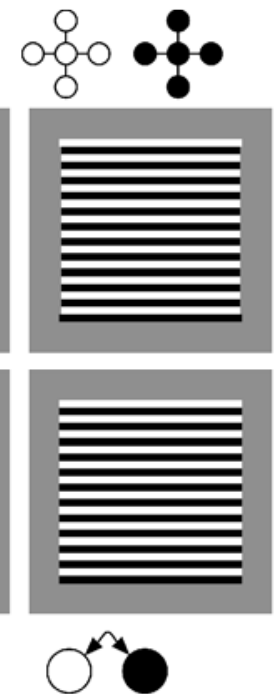
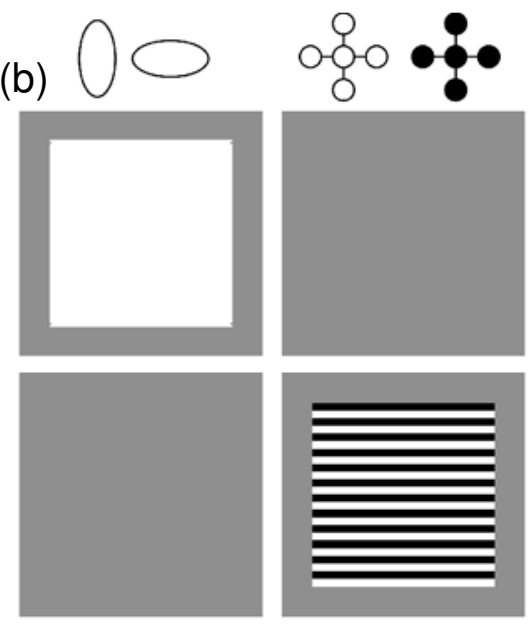

Image

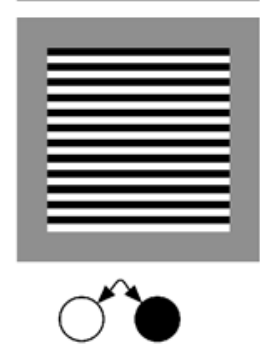

Figure 5. Simulation results describing the model's behavior during presentation and offset of a horizontal bar grating. (a) Model behavior during presentation of the grating. The image (lower left) is a black-and-white horizontal bar grating. The representation of black and white (lower right) is a copy of the image. The BCS (upper left) codes vertical (white) and horizontal (black) edges. The filling-in stages (upper right) are a veridical representation of the image. (b) Model behavior 1 sec after offset of the grating. The image (lower left) is blank gray. The representation of black and white (lower right) is a reverse contrast pattern of the original grating due to the afterresponses generated by the color gated dipole. The BCS (upper left) codes vertical (white) afterresponses. The filling-in stages (upper right) are a veridical copy of the blank gray image.

image. The frame on the top left represents activations of BCS boundary signals. The activity of different orientations is coded by color. A pixel colored middle gray indicates that neither a horizontally tuned nor a vertically tuned cell is responding at that pixel location. A white pixel indicates activity from a vertically tuned cell. A black pixel indicates activity from a horizontally tuned cell. The boundary representation of the BCS to the horizontal grating includes horizontal responses along the edges of the horizontal bars and vertical responses at the left and right sides of the grating, where there is contrast against the background.

The top right frame in Figure 5 a describes the activities of cells in the filling-in stages. The information is coded as for the frames in the bottom of Figure 5a, with white and light gray indicating responses among cells tuned to white, black and dark gray indicating responses among cells tuned to black, and middle gray indicating no response from either the white or black tuned cells. The calculation of the values is described in detail below. Intuitively, though, each pixel in the representation of color information (right frame on bottom row) feeds in to the pixel at the same position in the filling-in stage. An active cell in the filling-in stage then shares its activation with its neighbors, unless an appropriate BCS boundary signal is present. For example, at the pixel corresponding to the middle of the top bar of the horizontal grating, the fillingin stage receives a signal from the lower level that there is "white" at this location. That signal spreads (along with many others) left and right in the filling-in stage, but not up or down across the horizontal boundaries, which block it from spreading further. Likewise, the vertical boundaries at the left and right of the grating prevent the white signal from spreading left or right of the extent of the grating. A similar situation occurs for each black bar and white bar, so that the net effect is the seemingly uninteresting result that the filled-in percept is the same as the input image. More interesting filled-in percepts are generated when afterresponses are considered.

\section{Afterresponses in FACADE}

Grossberg (1991) and Francis, Grossberg, and Mingolla (1994) noted a computational problem with the use of excitatory feedback in the BCS. Although necessary to complete broken contours, the strong feedback also caused boundary signals to persist long after offset of the stimulus that generated them. Unless corrected, this persistence would lead to massive smearing of percepts. Following ideas outlined in Grossberg (1991), Francis et al. (1994) addressed this problem and demonstrated that the model's behavior explained properties of psychophysically measured visual persistence. The primary elaboration was to introduce a gated-dipole circuit (Grossberg, 1972), which creates afterresponses at offset of a stimulus. These afterresponses function as reset signals that break the excitatory feedback loop and thus shorten the duration of persisting signals in the loop. Figure 6a schematizes the type of gated-dipole circuit that was embedded in the BCS ar- 
(a)

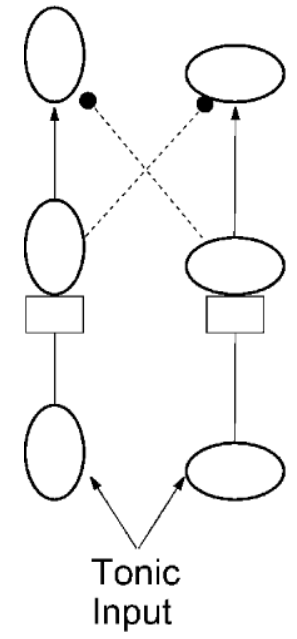

(b)

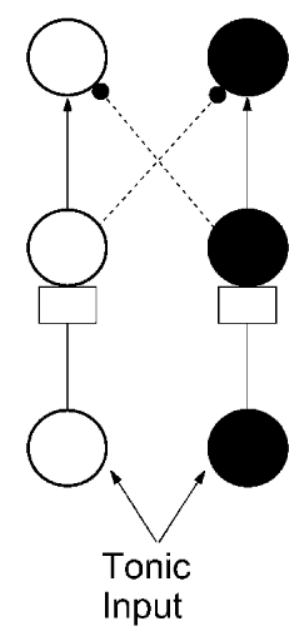

Figure 6. At stimulus offset, a gated-dipole circuit produces a transient rebound of activity in the nonstimulated opponent pathway. (a) An orientation gated dipole. (b) A color gated dipole.

chitecture. This circuit includes parallel channels that code for vertical and horizontal oriented edges. These orientation pathways compete with each other as signals pass from lower to higher levels of the circuit. Feeding this competition are inputs gated by habituative transmitter gates (schematized as boxes). At the offset of stimulation, a gated-dipole circuit produces a reduction in crosschannel inhibition from the stimulated channel to the unstimulated channel. This leads to a transient rebound of activity in the unstimulated pathway. In the overall design of the BCS, this rebound activity acts as a reset signal to curtail persisting neural signals. The properties of this gated dipole have been used to account for many properties of dynamic vision, including visual persistence (Francis, 1999; Francis et al., 1994) and motion perception (Francis \& Grossberg, 1996b; Francis \& Kim, 1999, 2001; Kim $\&$ Francis, 1998, 2000). Neurophysiological evidence has been found for gated dipoles in general (Abbot, Varela, Sen, \& Nelson, 1997) and for an orientation-based gated dipole in particular (Ringach, Hawken, \& Shapley, 1997).

Francis and Grossberg (1996a) noted that the behavior of oriented gated dipoles can account for properties of CAIs. Figure 7 shows the response of the BCS after offset of a vertical grating (as in Figure 5). The frame on the left shows the responses of the orientationally tuned cells that are the top layer in the gated dipole (Figure 6a). Each white pixel indicates an active vertical cell and each black pixel indicates an active horizontal cell. That the vertical white pixels are aligned in horizontal bars is a result of the stimulus that just turned off. That stimulus, the horizontal grating shown in Figure 5a, generated responses among a set of horizontally tuned cells. During their activation those cells habituated the transmitter gates in their respective gated dipoles. As a result, at offset of the grating,

orthogonally opposite responses are generated at exactly the same pixels.

The frame on the right shows the behavior of another set of cells that incorporates the excitatory feedback loop in the BCS. This loop is an interpolating process, whereby evidence of an oriented contour can generate responses among cells that interpolate the contour connecting the two sources of evidence. In the particular case shown in Figure 7, the responses of vertically tuned cells in each horizontal row initiate a feedback process that completes a vertical contour along each column. Thus, the white square in the frame on the right indicates a complete band of responses from vertically tuned cells. Such responses were taken by Francis and Grossberg (1996a) as corresponding to a CAI.

FACADE theory provides an explanation for differences between modal and amodal percepts. The modal percepts of surfaces, brightness, and colors correspond to activities in the filling-in stages of the FCS. However, amodal awareness of organization, shape, and some texture differences corresponds to the arrangement of boundary information in the BCS. CAIs are hypothesized to be the result of the afterresponses among cells in the BCS; this explains why CAIs are generally perceived as amodal. They do not necessarily have a color associated with them because the BCS does not represent color.

We now hypothesize that there is also a gated dipole between complementary colors (e.g., black and white), as schematized in Figure 6b. Mechanistically, this gated dipole works in exactly the same way as the orientation gated dipole shown in Figure 6a. In the color gated dipole, offset of white leads to an afterresponse of black, and vice versa. In the model, the color gated dipole is hypothesized to occur before, and to feed in to, the filling-in stages. It is schematized in Figure 4 as the curved dual arrow between the black cells and white cells. The output of the color gated dipole also feeds into the BCS, where it may influence orientation calculations.
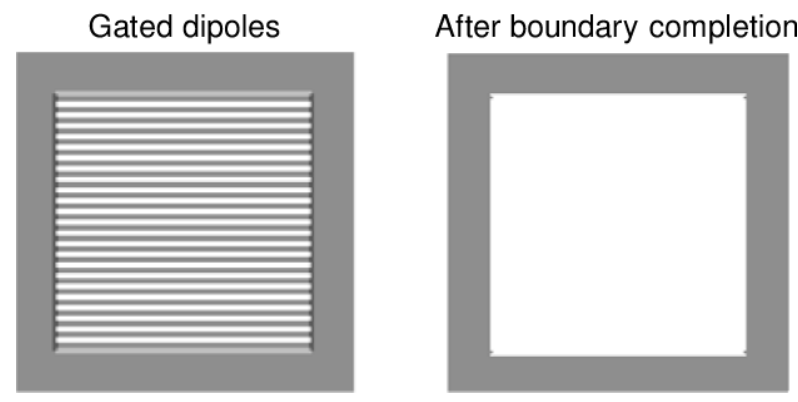

Figure 7. The behavior of two levels of the BCS at offset of a horizontal grating. The image on the left shows the behavior of the orientation gated dipole. The white lines indicate afterresponses among vertically tuned cells. The image on the right shows the BCS responses at the level of boundary completion. The solid white square indicates a dense array of responses among vertically tuned cells. The responses are generated by a grouping process that takes the signals from the gated dipole and completes signals in the spaces between. 
When both of the gated dipoles are included in FACADE processing, one sees an interesting interaction of the afterresponses. Figure $5 \mathrm{~b}$ shows the behavior of the BCS and FCS shortly after offset of the vertical grating in Figure 5a. In the simulation, the grating was presented for one simulated second, and the values shown in Figure 5b indicate the cell responses an additional second after the offset of the grating. The input image on the bottom left is of the gray background. The responses of the cells from the output of the color gated dipole are shown in the frame on the bottom right. Here the color gated dipole afterresponses produce what looks like a color negative of the original horizontal grating. All the locations that were previously stimulated by white now show afterresponses in the black channel, and vice versa.

The responses of the oriented cells show the effect of the oriented gated dipole and the completion effects of the bipole cells (as in Figure 7). The effect of these afterresponses is revealed in the frame of the filling-in stage responses (top right). The vertical boundary signals constrain color signals to only spread up or down. So along each column, white and black signals spread vertically. The net effect is that the black and white signals spread evenly across each column and cancel each other out. Thus, although the gray background may be seen, there is no percept of a black-and-white grating, even though it is present in the output of the color gated dipole. This agrees with the generally observed percept at offset of the presentation of a single grating.

Another way of describing the results in Figure $5 \mathrm{~b}$ is that the BCS input and the color input to the FCS have conflicting information about what the percept should be. The BCS is primarily representing vertically oriented signals, whereas the input to the FCS contains color information that is organized horizontally. In this mismatch of information, the constraints on filling-in imposed by the
BCS wash out the information proposed by the FCS. The next section demonstrates how FACADE accounts for the properties of MCAIs.

\section{Simulation of MCAIs}

This section demonstrates the creation of MCAIs in a simulation of FACADE. The discussion starts with the basic effect of MCAIs and then addresses each of the properties identified in the experiment.

Figure 8 shows the development of MCAIs by presenting the different components of the model during a simulated trial. The trial starts with the presentation of a horizontal black-and-white grating (Figure 8a). Here the output of the color gated dipole shows the input from the horizontal grating. The boundary signals are primarily horizontal, as induced by the grating. The filling-in stage shows a horizontal grating, and thus a veridical percept.

Figure $8 \mathrm{~b}$ shows the model's behavior when the horizontal grating is replaced by a vertical grating. As in the experiment, this vertical grating flickered with its color complement. Figure $8 \mathrm{~b}$ shows the behavior of the model at the end of the last vertical grating. The output of the color gated dipole shows predominately vertically arranged black and white color signals. However, faintly superimposed on the vertical pattern are black and white horizontal bars. (The faint horizontal stripes may not be visible in the reproduction of the image.) These are color afterresponses produced by the offset of the horizontal grating. The orientation signals are predominately vertical (white) because of two effects. First, the presentation of the vertical image produces strong responses among vertically tuned cells at the appropriate positions on the edges of the bars. Second, the offset of the horizontal grating has caused rebounds in the orientation gated dipole that produce strong vertical boundary responses. At the boundary completion stage, described in Figure 7, a dense block of (a)

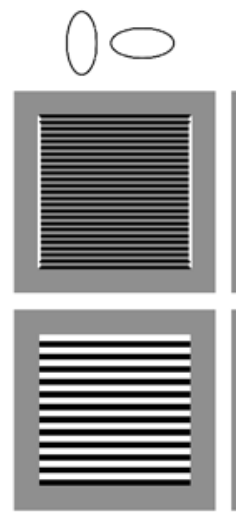

Image
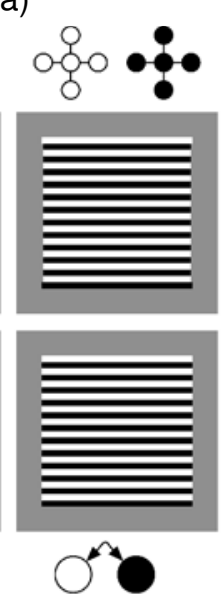

(b)
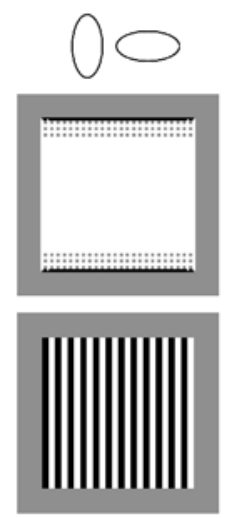

Image
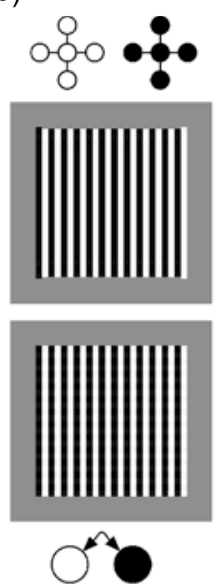

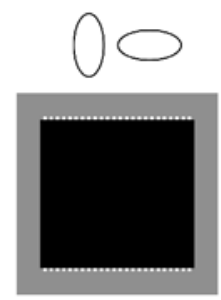

(c)
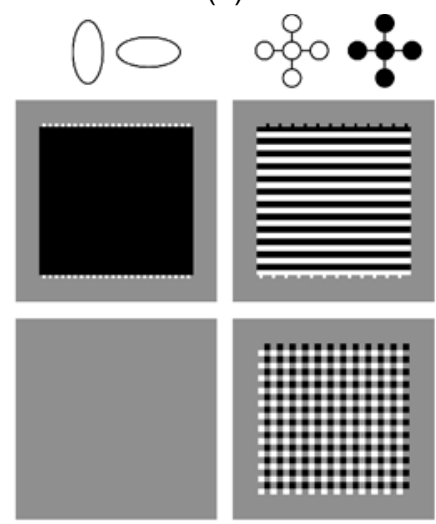

Image
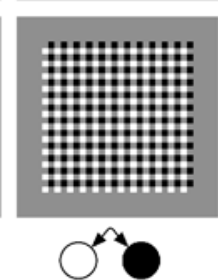

Figure 8. Simulation results for a sequence of images that produces MCAIs. (a) Model behavior during presentation of a horizontal grating. (b) Model behavior during the last of the flickering vertical bar gratings. (c) Model behavior $1 \mathrm{sec}$ after offset of the vertical bar grating. The filling-in stage shows a horizontal pattern, which corresponds to the MCAI. 
vertically tuned cells responds across the entire grating. The filling-in stage shows a vertical grating, which corresponds to a veridical percept.

Figure 8c shows the model's behavior $1 \mathrm{sec}$ after the offset of the vertical grating. The input image is a blank gray background. The responses of the color gated dipole produce a checkerboard pattern. This pattern is due to afterresponses generated by both the vertical and horizontal bar gratings. The orientation signals are primarily horizontal because offset of the vertical bar grating produces an afterresponse of horizontal signals. The filling-in stage shows a horizontal bar grating, which corresponds to the MCAI percept. The filling-in stage produces this pattern because the horizontal boundary signals constrain the filling-in signals to spread only left and right, not up and down. Thus, the dark and light columns of inputs from the color gated dipole spread across and cancel each other out. On the other hand, the dark and light rows in the color gated dipole are kept separate and so support activity at the filling-in stage. The net effect is that the orientation

(a)
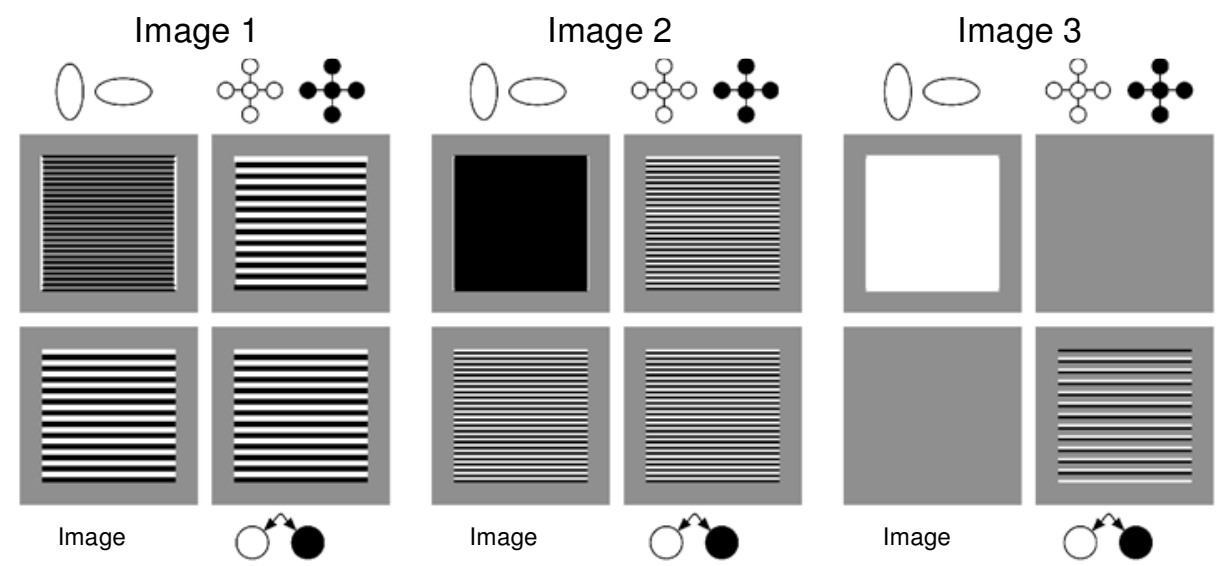

(b)
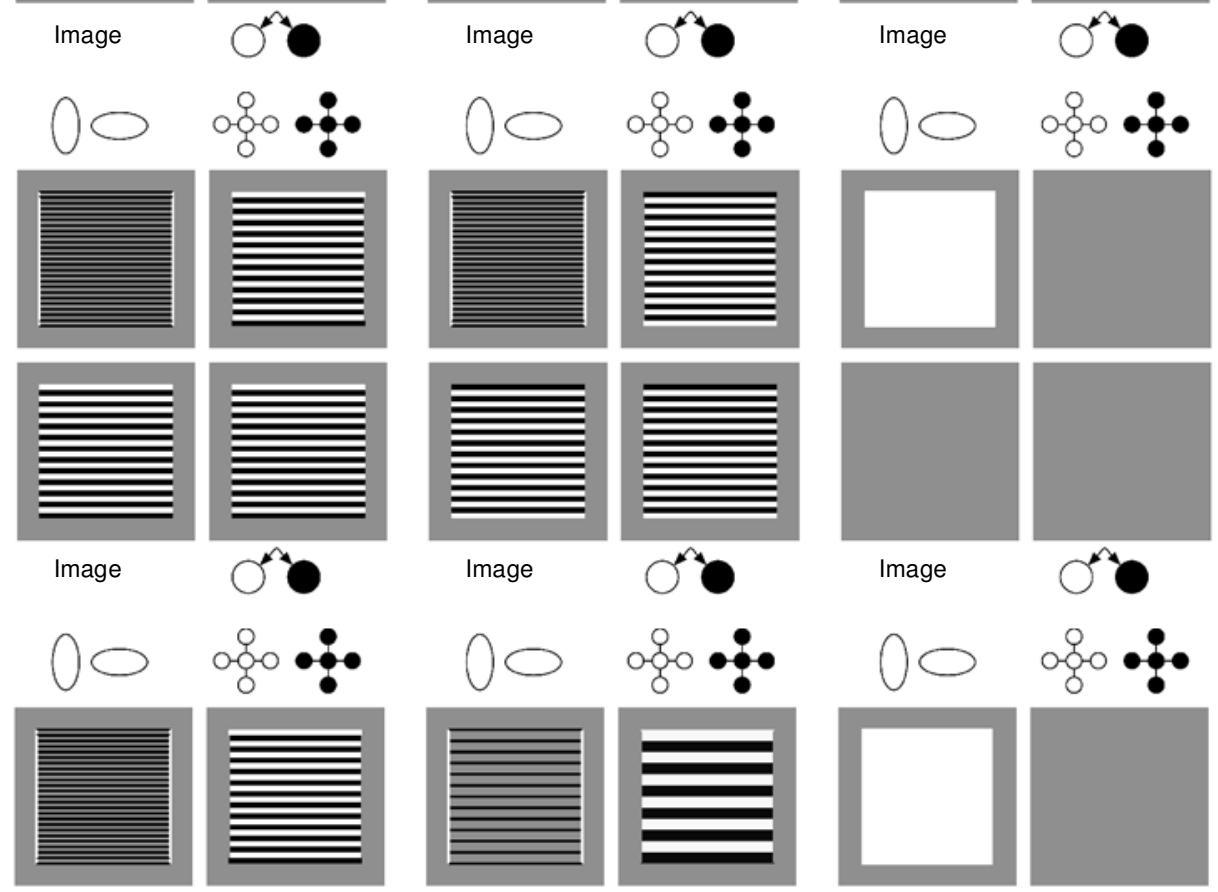

(c)
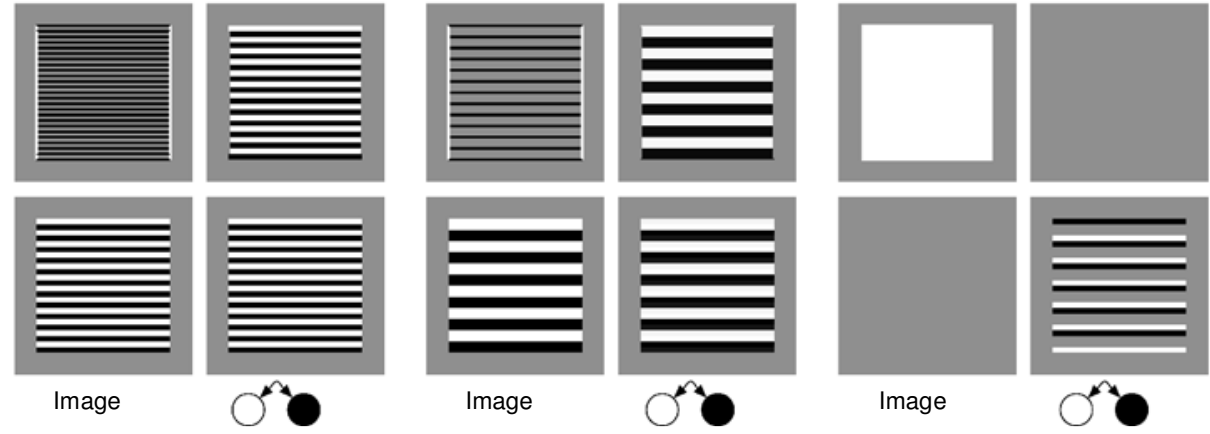

Image

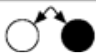

Figure 9. Simulation results when the second stimulus is a horizontal grating. No MCAIs are generated in the filling-in stage at the third frame. In (a) the second stimulus, bars are half the thickness of the first stimulus bars. In (b) the second stimulus has bars the same thickness as those of the first stimulus. In (c) the second stimulus, bars are twice the thickness of those of the first stimulus. 
Image 1

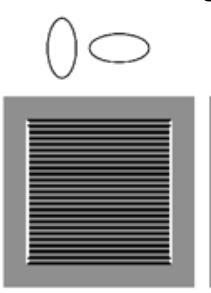

(a)

(b)

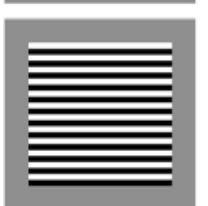

Image
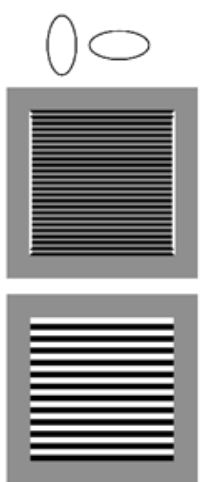

Image
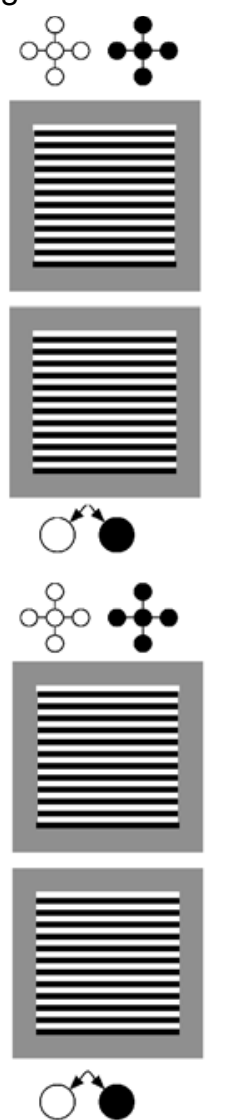

Image 2
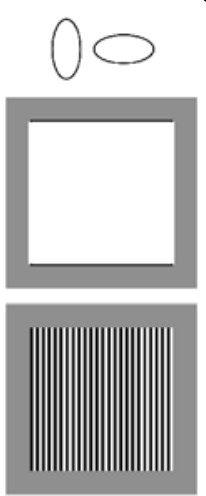

Image
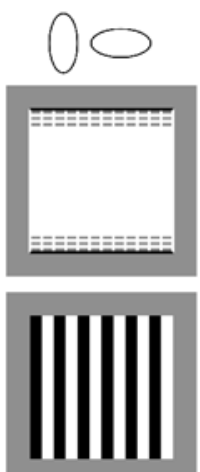

Image
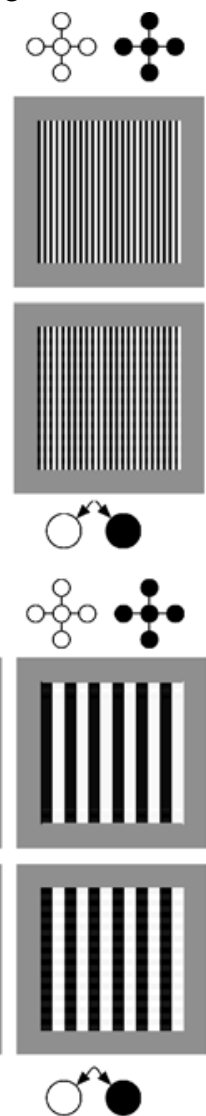
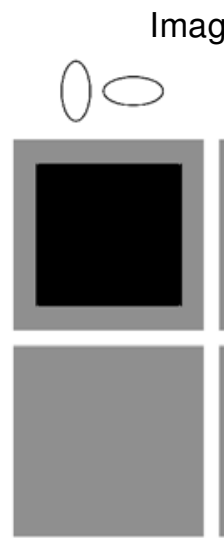

Image
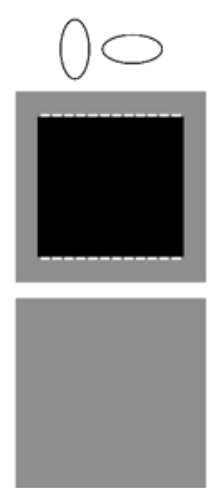

Image
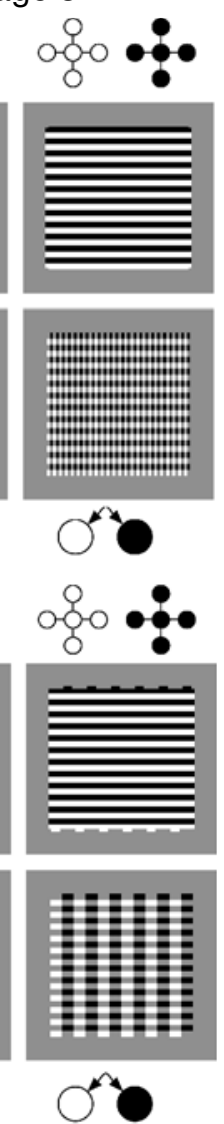

Figure 10. Simulation results when the second stimulus is a vertical grating, with different bar thicknesses than the first grating. MCAIs are generated in the filling-in stage at the third frame. In (a) the second stimulus, bars are half the thickness of the bars in the first stimulus. In (b) the second stimulus, bars are twice the thickness of the bars in the first stimulus.

afterresponses force the filling-in stages to "pick out" the horizontal pattern in the outputs of the color gated dipoles, thus giving the orientation-based CAI a modal percept. This simulation shows that the model produces the primary effect noted by Vidyasagar et al. (1999) and found in the experiment reported here.

Additional simulations show that the model accounts for the other effects noted in the experiment. Figure 9 shows simulation results when the second grating is horizontal rather than vertical. In Figure 9a, the second grating consists of bar elements half the size of the first grating. In Figure 9b, the second grating has bar elements the same size as the first grating. In Figure 9c, the second grating consists of bar elements twice the size of the first grating. In each condition, the first two images lead to veridical percepts of the presented stimuli. The most interesting effects are found for the third image. Regardless of the thickness of the second grating's bar elements, no MCAI is produced at the filling-in stage. A special case is when the second grating has bars the same thickness as the first grating. There is no response at the level of the color gated dipole. This occurs because the flickering sec- ond grating ends with a grating that is a color complement of the first grating. As a result, any residual afterresponses from the first grating are countered by afterresponses from the color complement stimulus. When the bar thickness of the second grating is different from the thickness of the bars in the first grating, the color gated dipole does show some afterresponses. However, these responses are always organized horizontally.

For all conditions, the orientation responses at the third frame are vertical (white) because offset of the horizontal stimuli leads to vertical afterresponses. These vertical orientation signals allow filling-in to occur only in vertical directions, not left or right. As a result, any activity at the level of the color gated dipole is washed out as it spreads vertically. This is consistent with the experimental finding that when the second grating has the same orientation as the first grating, observers do not report any type of afterimage.

Figure 10 shows simulation results when the second grating was vertical and had thin or thick bars. Consistent with the experimental findings, an MCAI is produced regardless of the thickness of the second grating's bars. Moreover, the thickness of the bars in the simulation's 
(a)
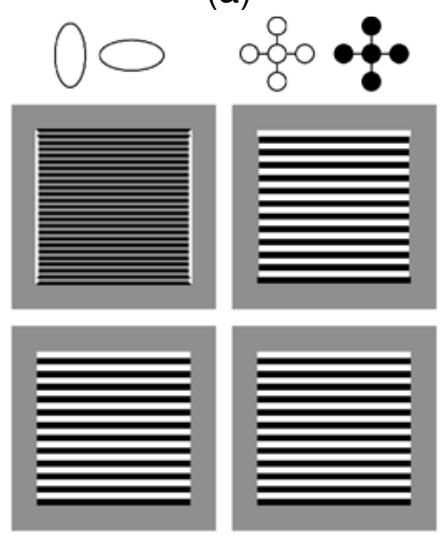

Image

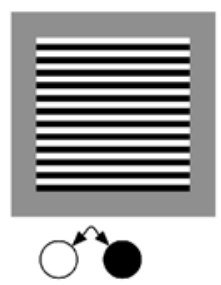

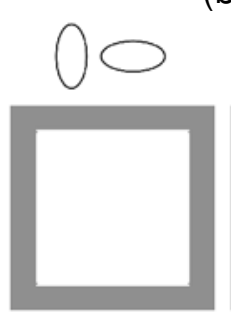

(b)
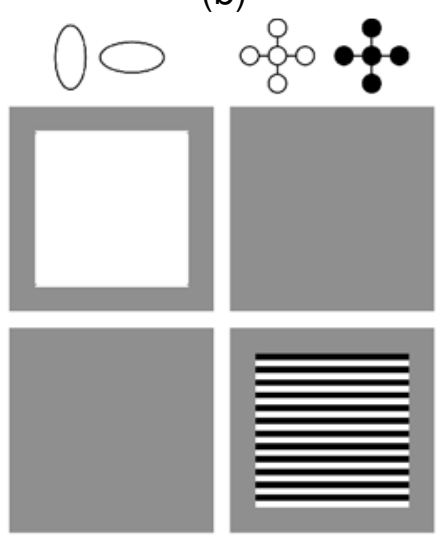

Image

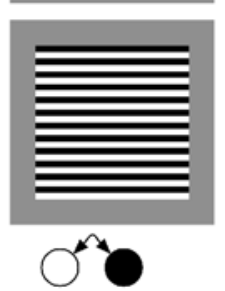

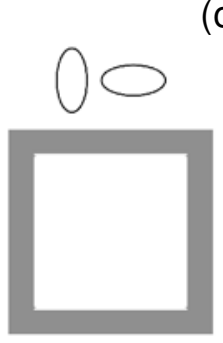

(c)

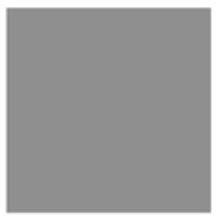

Image
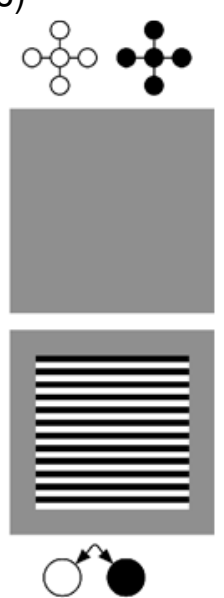

Figure 11. Simulation results when the second stimulus is a blank. (a) Model behavior during presentation of a horizontal grating. (b) Model behavior at the end of the presentation of a 1-sec blank grating. (c) Model behavior 1 sec later. No MCAI is produced.

MCAI corresponds to the thickness of the bars in the first grating, not the bars of the second grating. Although this was not measured in the experiment, our own observations of the MCAI afterimage agree with this model prediction.

Finally, we turn to the model's behavior when the second image is blank. Figure 11 shows the model's behavior for this condition. In agreement with the reports of most of the observers in the experiment, the filling-in stage does not represent any type of afterimage percept. In this condition, the color gated dipole does produce a set of afterresponses that would support a horizontal afterimage, but the afterresponses are weak and so cannot generate a boundary representation in the BCS that will support the horizontally arranged color signals at the filling-in stage. Indeed, the orientation gated dipole produces vertical afterresponses, which have the effect of washing out the color inputs to the filling-in stage.

The experimental data show observer differences when the second stimulus was blank. Three of the 7 observers reported seeing a horizontal afterimage at least $50 \%$ of the time. The model can accommodate these observer differences with a change in parameters. Figure 12 shows the model's behavior when the parameters were changed so that the color gated dipole produced stronger afterresponses. Figure 12a shows the model's behavior when the second stimulus was blank. Because the color gated dipole produces stronger afterresponses, it is able to generate horizontal boundary representations in the BCS and thus support a negative afterimage at the filling-in stage.

Figure $12 \mathrm{~b}$ shows the model with its modified parameters in response to the traditional MCAI-inducing sequence of images. Although there are minor differences, the basic MCAI is observed. Thus, the model accounts for the experimental finding that the visibility of the MCAI is not directly related to the visibility of a negative afterimage.

Interestingly, with its modified parameters, the model predicts that when viewing the vertical grating the ob- server should see a faint superimposed horizontal afterimage mingled with the percept of the vertical grating. (The faint horizontal stripes in the filling-in stage of Figure $12 \mathrm{~b}$ may not be visible in the reproduction of the image.) Although we did not ask our observers to report on this type of percept, in pilot work some observers commented that they saw such percepts.

\section{CONCLUSIONS}

We have provided experimental data about the appearance of the type of afterimage first reported by Vidyasagar et al. (1999). Our data suggest that the MCAI is very much dependent on the orientation of the second stimulus. At least over the range of stimuli we used, the MCAI does not seem to be dependent on the spatial frequency of the second stimulus. It is not necessary for the inducing stimuli to be moving. Finally, the MCAI does not seem to be directly related to the presence of a negative afterimage that might be seen after presentation of a single grating.

Our simulations of the FACADE model (Grossberg, 1994, 1997; Grossberg \& Mingolla, 1985a, 1985b) suggest that it can account for all of these properties of MCAIs. The primary model characteristics related to the appearance of MCAIs are as follows: a boundary system that produces orientation afterresponses, a color system that produces color afterresponses, and a filling-in system that combines the color and boundary information to form a surface representation. We believe that any system that hopes to account for the properties of MCAIs will need these minimal components. Our analysis of the FACADE theory substantially refines the explanation of MCAIs that was proposed by Vidyasagar et al. (1999). Although their explanation included cross-orientation inhibition and a fading trace that corresponded to the first inducing stimulus, they did not consider the importance of distinguishing between boundary and surface mechanisms or the crit- 
(a)

(b)

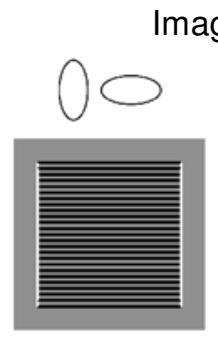

Image 1

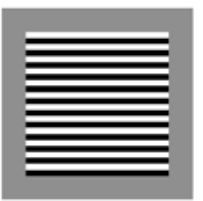

Image
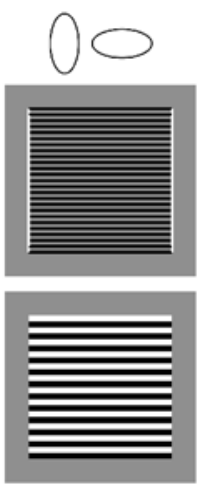

Image
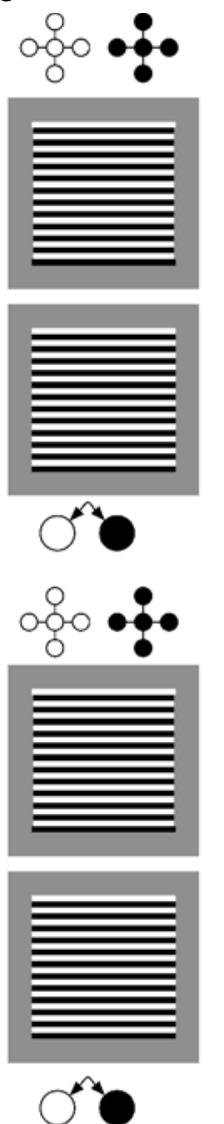

Image 2
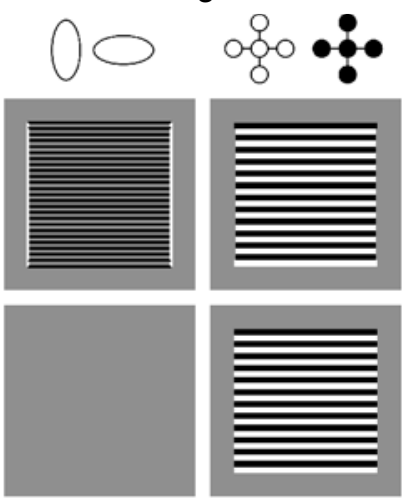

Image
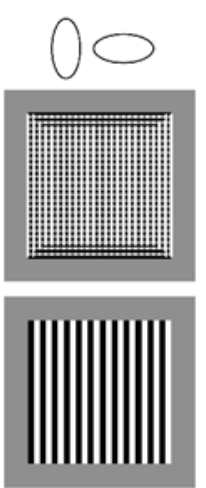

Image
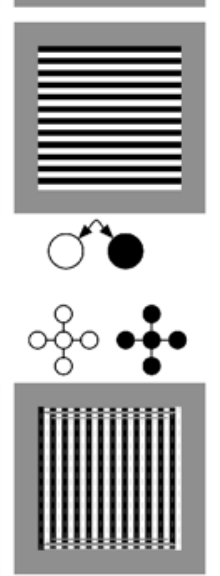
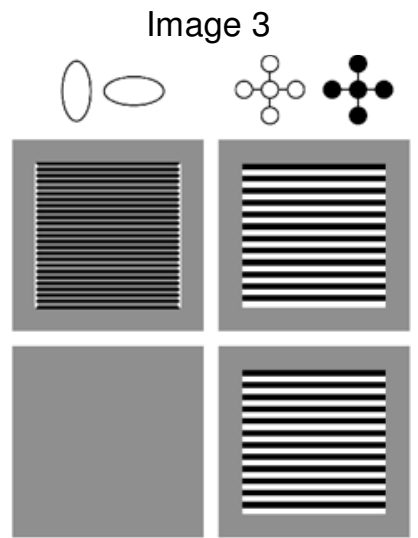

Image
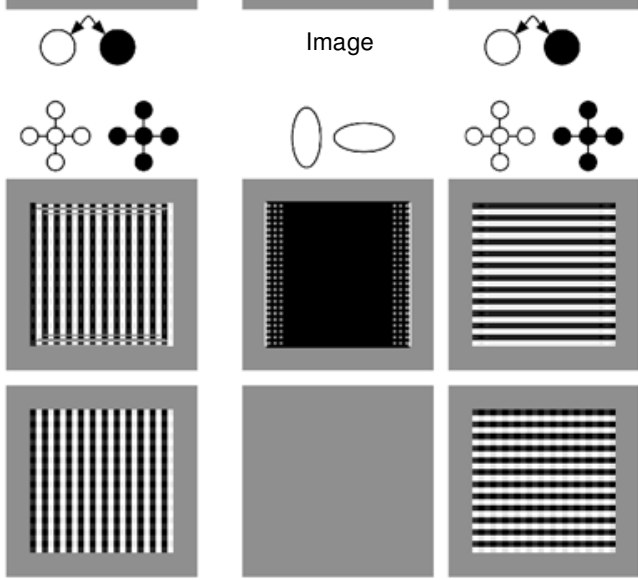

Image

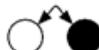

Figure 12. Simulation results with an alternative set of parameters that produce stronger color gated dipole afterresponses. In (a) the second stimulus was a blank gray screen. The model produces a color complement afterimage after offset of the horizontal grating. This persists for at least two 1-sec frames. In (b) the second stimulus was a vertical grating. The standard MCAI is seen at offset of the vertical grating.

ical role that filling-in mechanisms play in accounting for the appearance of MCAIs.

We also emphasize that we have added no new components to the FACADE theory. All of the necessary components existed in the model before the properties of MCAIs were observed. Thus, the results presented here do not just demonstrate that simulations of the FACADE theory can be made to match the percepts of MCAIs. Instead, the simulations connect the MCAI percepts to specific mechanisms and properties of the theory. As a result, the theory offers an explanation about why MCAIs are observed at all. Namely, the theory says that MCAIs are a side effect of neural mechanisms that are necessary to support the proper computation of surface and boundary features in the visual system.

From this perspective, if MCAIs were not observed, or had characteristics much different from what have so far been discovered, the FACADE theory would need dramatic modification. Thus, we believe that further exploration of MCAIs will allow us to identify detailed properties of the boundary and surface mechanisms that we believe are re- sponsible for the MCAI percepts. This type of afterimage may become a valuable tool for exploring the computational and neurophysiological details of filling-in phenomena.

\section{REFERENCES}

Аввот, L. F., Varela, J. A., Sen, K., \& Nelson, S. B. (1997). Synaptic depression and cortical gain control. Science, 275, 220-224.

Blakemore, C., \& Sutton, P. (1969). Size adaptation: A new aftereffect. Science, 166, 245-247.

Francis, G. (1999). Spatial frequency and visual persistence: Cortical reset. Spatial Vision, 12, 31-50.

Francis, G., \& Grossberg, S. (1996a). Cortical dynamics of boundary segmentation and reset: Persistence, afterimages and residual traces. Perception, 25, 543-567.

Francis, G., \& Grossberg, S. (1996b). Cortical dynamics of form and motion integration: Persistence, apparent motion, and illusory contours. Vision Research, 36, 149-174.

Francis, G., Grossberg, S., \& Mingolla, E. (1994). Cortical dynamics of binding and reset: Control of visual persistence. Vision Research, 34, 1089-1104.

FrancIs, G., \& KIM, H. (1999). Motion parallel to line orientation: Disambiguation of motion percepts. Perception, 28, 1243-1255.

Francis, G., \& KIM, H. (2001). Perceived motion in orientational afterimages: Direction and speed. Vision Research, 41, 161-172. 
Gibson, J. J. (1933). Adaptation, after-effect, and contrast in the perception of curved lines. Journal of Experimental Psychology, 16, 131.

Grossberg, S. (1972). A neural theory of punishment and avoidance: II. Quantitative theory. Mathematical Biosciences, 15, 253-285.

Grossberg, S. (1991). Why do parallel cortical systems exist for the perception of static form and moving form? Perception \& Psychophysics, 49, 117-141.

Grossberg, S. (1994). 3-D vision and figure-ground separation by visual cortex. Perception \& Psychophysics, 55, 48-120.

GrossBerg, S. (1997). Cortical dynamics of three-dimensional figureground perception of two-dimensional figures. Psychological Review, 104, 618-658.

Grossberg, S., \& Kelly, F. J. (1999). Neural dynamics of binocular brightness perception. Vision Research, 39, 3796-3816.

Grossberg, S., \& McLoughlin, N. (1997). Cortical dynamics of 3-D surface perception: Binocular and half-occluded scenic images. Neural Networks, 10, 1583-1605.

Grossberg, S., \& Mingolla, E. (1985a). Neural dynamics of form perception: Boundary completion, illusory figures, and neon color spreading. Psychological Review, 92, 173-211.

Grossberg, S., \& Mingolla, E. (1985b). Neural dynamics of perceptual grouping: Textures, boundaries, and emergent segmentations. Perception \& Psychophysics, 38, 141-171.
Grossberg, S., Mingolla, E., \& Ross, W. D. (1997). Visual brain and visual perception: How does the cortex do perceptual grouping? Trends in Neurosciences, 20, 106-111.

Grossberg, S., \& Todorović, D. (1988). Neural dynamics of 1-D and 2-D brightness perception: A unified model of classical and recent phenomena. Perception \& Psychophysics, 43, 241-277.

KANIZSA, G. (1979). Organization in vision: Essays on Gestalt perception. New York: Praeger.

KIM, H., \& FRANCIS, G. (1998). A computational and perceptual account of motion lines. Perception, 27, 785-797.

KIM, H., \& Francis, G. (2000). Perceived motion in complementary afterimages: Verification of a neural network theory. Spatial Vision, 13, 67-86

KöHLER, W., \& WALLACH, H. (1944). Figural after-effects: An investigation of visual processes. Proceedings of the American Philosophical Society, 88, 269-357.

MACKAY, D. (1957). Moving visual images produced by regular stationary patterns. Nature, $\mathbf{1 8 0}, 849-850$.

McCollough, C. (1965). Color adaptation of edge-detectors in the human visual system. Science, 149, 1115-1116.

Ringach, D., HaWken, M. J., \& Shapley, R. (1997). Dynamics of orientation tuning in macaque primary visual cortex. Nature, 387, 281-283.

Vidyasagar, T. R., BuZAs, P., Kisy ARday, Z F., \& Ey SEL, U. T. (1999). Release from inhibition reveals the visual past. Nature, 399, 422-423.

\section{APPENDIX \\ Simulations}

The model simulations are a simplification and modification of the BCS and FCS simulations described in Grossberg and Mingolla (1985a, 1985b) and Grossberg and Todorović (1988). More complete and neurophysiologically based simulations of FACADE can be found in Grossberg and Kelly (1999), Grossberg and McLoughlin (1997), and Grossberg, Mingolla, and Ross (1997).

\section{Image Plane: Input image}

Each pixel $(i, j)$ had an input value $I_{i, j}$. All the images consisted of three colors (white, 1 ; black, -1 ; and gray, $0)$. A $128 \times 128$ pixel plane was used in all simulated images. A bar grating image was always centered on the image plane and had a size of $96 \times 96$ pixels. The thickness of each black or white bar was 2,4 , or 8 pixels, for the thin, medium, and thick bars, respectively.Each image was presented for 10 simulated time units, which corresponds to $1 \mathrm{sec}$ of presentation in the experiment. Each image plane had its origin at the upper left corner. Larger positive indices indicate locations to the right or below the origin.

\section{Color Gated Dipole}

The $I_{i, j}$ value at each pixel fed directly into a color gated dipole. Here black and white signals were sent through opponent channels, and habituation of those channels occurred. The habituating gate for the white channel at pixel $(i, j), g_{i, j}$ obeyed the following differential equation:

$$
\frac{d g_{i, j}}{d t}=\left[A-B g_{i, j}-C g_{i, j}\left(\left[I_{i, j}\right]^{+}+J\right)\right] D .
$$

The term $A-B g_{i, j}$ describes a process whereby the gate amount increases toward the value $A / B$. The last subtraction describes depletion of the gate by the presence of a tonic signal $J$ and by a white signal, $\left[I_{i, j}\right]^{+}$, where the notation [ ] $]^{+}$indicates that negative values are set to zero. Parameter $D$ controls the overall rate of change of the equation.

The black opponent pathway had an identical type of equation with only the term $\left[I_{i, j}\right]^{+}$replaced by $\left[-I_{i, j}\right]^{+}$, so that only black signals passed through the gate. In the following equations the habituating gate for the black pathway is labeled as $G_{i, j}$. Each simulation trial started with an initial value of gates that corresponded to an equilibrium value of the gates with no outside input:

$$
g_{i, j}(0)=G_{i, j}(0)=\frac{A}{B+C J} .
$$

The parameters were set as $A=B=C=1, D=0.1$, and $J=0.5$.

The white output of the color gated dipole was then computed by weighting the net input into the white channel by its gate and then subtracting the resulting value by the weighted black input. The resulting difference was thresholded and then scaled. The white output, $w_{i, j}$, was computed as

$$
w_{i, j}=E\left[\left(\left[I_{i, j}\right]^{+}+J\right) g_{i, j}-\left(\left[-I_{i, j}\right]^{+}+J\right) G_{i, j}-F\right]^{+} .
$$


APPENDIX (Continued)

Here, $F=0.004$ is a threshold, the term to its left is the inhibitory input from the black channel, and the next term to the left is the excitatory input from the lower levels of the white channel. The difference is rectified so that negative values are set to zero and then scaled by the multiplying term $E=100$. The black output of the gated dipole, $b_{i, j}$, had a similar equation, with only the middle and left terms trading excitatory and inhibitory roles. The value $w_{i, j}-b_{i, j}$ was plotted in the simulation figures (bottom right frame).

\section{Boundary Contour System}

\section{Edge Detection and Orientation Gated Dipole}

The set of $w_{i, j}$ and $b_{i, j}$ values was sent to the BCS for edge detection. At each pixel, detectors were sensitive to an activation pattern that signaled either a vertical or a horizontal edge. For computational simplicity, exceedingly simple detectors were used that just looked for a change in luminance intensity in a vertical or horizontal direction. The absolute value of this change was taken as the response of the detector. A boundary cell at position $(i, j)$ tuned to a vertical edge had an activity

$$
y_{i, j}=\left[\left|w_{i, j}-w_{i-1, j}\right|+\left|w_{i, j}-w_{i+1, j}\right|+\left|b_{i, j}-b_{i-1, j}\right|+\left|b_{i, j}-b_{i+1, j}\right|-K\right]^{+} .
$$

This receptive field looks to the left and right of the edge location for any discontinuities among the white or black representations of color. The term $K=20$ indicates a threshold. Any values below $K$ were set to zero. A similar value, $Y_{i, j}$, was computed for the horizontally tuned boundary cells.

The equations for the BCS-oriented gated dipole were identical in form to those used for the color gated dipole. The only differences from the equations of the color gated dipole are that in the BCS version of Equations A1 and $\mathrm{A} 3,\left[I_{i, j}\right]^{+}$is replaced by $y_{i, j}$ (vertical), $\left[-I_{i, j}\right]^{+}$is replaced by $Y_{i, j}$ (horizontal), $D=1.0, E=1.0$, and $F=$ 0.0. The relevant equations are as follows:

$$
\frac{d g_{i, j}}{d t}=\left[A-B g_{i, j}-C g_{i, j}\left(y_{i, j}+J\right)\right] D
$$

and

$$
x_{i, j}=E\left[\left(y_{i, j}+J\right) g_{i, j}-\left(Y_{i, j}+J\right) G_{i, j}-F\right]^{+} .
$$

Here, $x_{i, j}$ refers to the output of the orientation gated dipole for vertically tuned cells. Since the outputs of the color gated dipole feed into the orientation detectors, which in turn feed into the habituative gates for the orientation gated dipole, the differentialequations for both sets of the habituative gates were solved simultaneously.

\section{Boundary Grouping}

Grouping of BCS signals is supported by model bipole cells. Each vertically tuned bipole cell received excitation from vertically tuned gated dipole output cells and received inhibition from horizontally tuned gated dipole output cells. A vertical bipole cell had two sides (Up and Down) that separately combined excitatory and inhibitory inputs from the lower level. Each side combined information from a column of locations above (Up) or below (Down) the location of the bipole cell. For a vertical bipole cell at position $(i, j)$, define intermediate terms:

$$
\mathrm{Up}_{i, j}=\left[\sum_{k=2}^{10}\left(x_{i, j-k}-X_{i, j-k}\right)\right]^{+}
$$

and

$$
\operatorname{Down}_{i, j}=\left[\sum_{k=2}^{10}\left(x_{i, j+k}-X_{i, j+k}\right)\right]^{+}
$$

where $x_{i, j}$ and $X_{i, j}$ refer to the output of the orientation gated dipole for vertically and horizontally tuned cells, respectively. A bipole cell has a positive activity if both of the intermediate terms are nonzero, or the bottomup edge information at the bipole cell's pixel location is nonzero and one of the intermediate terms is also nonzero. Thus, the activity of the vertical bipole cell at pixel $(i, j)$ is as follows:

$$
B_{i, j}=\max \left(\mathrm{Up}_{i, j} \operatorname{Down}_{i, j} ; x_{i, j} \mathrm{Up}_{i, j} ; x_{i, j} \operatorname{Down}_{i, j}\right) .
$$

This allows bipole cells to respond as if they interpolate, but do not extrapolate, bottom-up contour information. A vertical bipole cell will also respond if an oriented edge is present at the location of the bipole cell and a vertical oriented edge is above or below the location of the bipole cell. Similar equations exist for each horizontally tuned bipole cell, which received excitatory input from other horizontally tuned cells and inhibitory input from vertically tuned cells.

To avoid proliferation of bipole cell responses, a second competition between orientations at each pixel was included. This competition was winner-take-all, so that the orientationbipole cell with the largest value received a value of one, and the other orientation bipole cell was given a value of zero. The set of values of each orien- 
APPENDIX (Continued)

tation at each pixel location was taken as the "output" of the BCS. A pixel was said to have a vertical boundary if the vertically tuned cell won the competition here. A more elaborate version of this type of competition can be found in Grossberg et al. (1997).

\section{Feature Contour System}

In the present simulations, the FCS consists only of the filling-in stages. There are separate filling-in stages for white and black. We will describe the calculations for the white filling-in stage. The equations are identical for the black filling-in stage, with only the terms for black and white switched. Each pixel in a filling-in stage has separate activity values for the white and black filling-in stages.

To implement the filling-in process, the simulation proceeded in an algorithmic way. Similar results are found with a diffusive process (e.g., Grossberg \& Todorović, 1988) that could easily be instantiated in the nervous system.

The first step in the filling-in process was to identify connected surfaces or Filling-In DOmains (FIDOs). This was done by assigning each pixel in an image plane a unique value. Then each pixel was sampled in turn and each of its nearest neighbors was examined to see which had a larger value. After each comparison, both pixels were assigned the larger value. The comparison did not occur across pixels separated by an appropriate boundary. Neighbors above or below the sampled pixel were not compared if either the sampled or the neighbor pixel had a horizontal boundary signal. Likewise, neighbors right or left of the sampled pixel were not compared if either the sampled or the neighbor pixel had a vertical boundary signal. Additionally, the comparison did not occur among vertical neighbors unless both the sampled and neighbor pixel both had vertical boundaries or neither had vertical boundaries. Among horizontal neighbors, the comparison did not occur unless both pixels had horizontal boundaries or neither had horizontal boundaries.

The comparisons were iterated across the entire image plane until there were no further changes in any pixel values. At the end of the iteration process, the pixel plane was split into regions with unique pixel values. These are the FIDOs. The effect of the above-mentioned comparison rules was to connect into a single FIDO pixels that are either connected by a path with no intervening boundary or are connected by a set of continuous horizontal or vertical contours.

The activity of the white filling-in stage is then found by calculating the average difference between the white inputs and black inputs across each separate FIDO. This value was then assigned to every cell in the FIDO. Positive values were taken as indicating white, and negative values were taken as indicating black. These values were normalized and used to create the filling-in results shown in the simulation figures.

\section{Alternative Parameters}

For the simulations in Figure 12, the following parameters were changed. For the color gated dipole, $A=10$, $D=0.05$, and $F=0.0004$. The threshold for oriented detectors was set to $K=1$ and for the oriented gated dipole, $D=5.0$. 